\title{
Agro-morphological Characterization of Bangladeshi Aromatic Rice (Oryza sativa L.) Germplasm Based on Qualitative Traits
}

\author{
M Z Islam ${ }^{1 *}$, M Khalequzzaman ${ }^{1}, \mathrm{M} \mathrm{K}$ Bashar $^{2}$, N A Ivy³, M M Haque ${ }^{3}$, \\ M A K Mian ${ }^{3}$ and M Tomita ${ }^{4}$
}

\begin{abstract}
The agro-morphological characterization of germplasm is of utmost importance to generate information to be utilized in plant breeding programmes. The aim of this study was to characterize the agro-morphological traits of 113 accessions of aromatic germplasm (Oryza sativa L.) based on qualitative agro-morphological descriptors. No duplicates were identified among the studied accessions for qualitative traits in the cluster analysis, which means there is a high diversity among the accessions for these traits. Following UPGMA cluster analysis, 113 accessions of aromatic germplasm formed ten distinct clusters. The highest numbers of germplasm (96) were found in cluster IXd, 2 were found in cluster III, IV and VI, 3 were found in IXc and the lowest number of germplasm (1) in cluster I, II,V, VII, VIII, IXa, IXb and X, respectively. Aroma evaluation revealed that 67 germplasm were scented, 34 were lightly scented, while the rest 12 germplasm were nonscented. Germplasm namely Begun bichi, Elai, Chinigura, Basmati 370, Ranisalut, Sakkorkhora, Jirakatari, Raduni Pagal, Kalijira (long grain), Black TAPL-554, Kalgochi, BRRI dhan34, BRRI dhan50, Badshabhog-2, Tulsimala-2, Kataribhog, BU dhan2R, Sakkorkhana, Maloti, Bashful could be used for further improvement for incorporating aroma to the high yielding varieties.

Keywords: Agro-morphological characterization, aromatic rice germplasm, qualitative traits
\end{abstract}

\section{INTRODUCTION}

Bangladesh is mainly a country of rice based cropping system, where thousands of local rice varieties are being cultivated from the time immemorial. Still now, farmers are cultivating local landraces in most of the unfavourable ecosystems. Traditional varieties have some special characteristics such as aroma, taste and better cooking quality, which also provide additional value in socio-economic aspects. Moreover, aromatic rice germplasm constitutes a special group of rice genotypes well known in many countries of the world for their aroma and or super fine grain quality (Singh et al., 2000, Islam et al., 2013). The Himalayan foothills including parts of Bangladesh are considered to be the secondary centre of diversity of the genus oryza (Morishima, 1984). Bangladesh has a stock of above 8,500 rice germplasm of which around 100 are aromatic genotypes (Islam et al., 2018a). The Bangladeshi aromatic and fine rice germplasm is comprised of short and medium bold types with mild to strong aroma (Shahidullah et al., 2009; Islam et al., 2016). Since the time of civilization, thousands of locally adapted aromatic rice genotypes have evolved as a consequence of natural and human selection. These landraces are the genetic reservoirs of useful genes. The large scale spread of modern, high yielding varieties have replaced the traditional varieties especially in the irrigated rice ecosystem leading to reduced genetic base and thus increased genetic vulnerability. Therefore, rice germplasm need

\footnotetext{
${ }_{1}^{1}$ Genetic Resources and Seed Division, Bangladesh Rice Research Institute (BRRI), Gazipur. 2Country Manager, CIAT, Harvest plus, Bangladesh. ${ }^{3}$ Professor, Bangabandhu Sheikh Mujibur Rahman Agricultural University (BSMRAU), Gazipur. ${ }^{4}$ Laboratory Genetics and Genomics, Department of Bioscience, Graduate School of Science and Technology, Shizuoka University, 836 Oya, Suruga-ku, Shizuoka, Shizuoka 422-8529. "Corresponding author's E-mail: zahid.grs@gmail.com
} 
to be utilized for maintaining its diversity in the field.

Agro-morphological characterization of germplasm accessions is essential in order to offer information for plant breeding programmes (Nascimento et al., 2011). Several researchers reported the use of agromorphological markers in the characterization and study of rice (Oryza sativa L.) germplasm diversity (Islam et al., 2017; Mau et al., 2017; Akter et al., 2018). Aromatic rice varieties in general are tall statured, possess fewer number of panicles, high stem weight, lower yields and susceptible in lodging (Islam et al., 2016). Glaszmann (1987) reported that aromatic rice germplasm fall into a separate group from that of the typical indicas and declared that these two groups are incompatible causing intergroup hybrid sterility. Recently it is revealed that 2-acetyl-1-pyrroline based fragrance in rice is due to the presence of a non-functional betaine aldehyde dehydrogenase 2 (BADH2) (Bradbury et al., 2005, 2008). The nonfunctional $\mathrm{BADH} 2$ interferes in pollen tube development and this could be the cause for the low grain yield in aromatic germplasm (Bradbury et al., 2008). Morphological characterization is the first step in the classification and assessment of the germplasm. Although large number of germplasm collections is known to exist in BRRI Genebank in Bangladesh, not all of them have been fully and properly characterized and documented. Therefore, systematic attempts have to be taken to make a total inventory of this valuable gene pool for quantifying the availability of new useful genes of this source. Besides, it is very important to protect bio-piracy and geographical indications and issues related Intellectual Property Rights (IPR). On the other hand, researches on qualitative traits evaluation on aromatic rice germplasm are almost nil. Considering the above fact, the present study was initiated to characterize the qualitative agro-morphological characters of aromatic germplasm of Bangladesh.

\section{MATERIALS AND METHODS}

\section{Experimental site and plant materials}

The experiment was conducted at the farm of Bangladesh Rice Research Institute (BRRI), Gazipur in T. Aman season, 2011. A total of 113 aromatic germplasm were evaluated using "Germplasm Descriptors and Evaluation Form" approved by BRRI (Table 1). Names for the 113 aromatic rice germplasm along with methods have been previously described by Islam et al. (2016).

\section{Agro-morphological traits observation}

We observed variables of 28 qualitative agromorphological characters namely: 1 . Blade pubescence, 2. Blade colour, 3. Leaf sheath: anthocyanin colour (early to late vegetative stage), 4 . Basal leaf sheath colour (early to late vegetative stage), 5 . Leaf angle (prior to heading), 6. Flag leaf angle (after heading), 7. Ligule colour (late vegetative stage), 8. Ligule shape (late vegetative stage), 9. Coller colour (late vegetative stage), 10. Auricle colour (late vegetative stage), 11. Culm: anthocyanin colouration of nodes (after flowerting), 12. Culm angle (after flowerting), 13. Internode colour (after flowering), 14. Culm strength (after flowerting to maturity), 15. Panicle type (near maturity), 16. Secondery branching (near maturity), 17. Panicle exsertion (near maturity), 18. Spikelet: awns in the spiklet, 19. Spikelet: length of the longest awn (flowering to maturity), 20. Distribution of awning (flowering to maturity), 21. Awn colour (at maturity), 22. Apiculus colour (at maturity), 23. Stigma colour (at flowering), 24. Lemma and palea colour (at maturity), 25. Lemma and palea pubescence (at maturity), 26. Seed coat colour (at maturity), 27. Leaf senescence (at maturity), 28. Decorticated grain: scent (aroma), at maturity stage. The observed qualitative traits were scored based on "Germplasm Descriptors and Evaluation Form" issued by BRRI prior to data analysis (Table 2). 
Table 1. List of $\mathbf{1 1 3}$ aromatic germplasm used in morphological characterization.

\begin{tabular}{|c|c|c|c|c|c|}
\hline Germplasm & Acc. No. & District/Source & Germplasm & Acc. No. & District/Source \\
\hline Sakor & 197 & Mymensingh & Khasa & 682 & Cumilla \\
\hline Sagardana & 229 & Mymensingh & Buchi & 369 & Gaibandha \\
\hline Nunia & 233 & Mymensingh & Awned TAPL-545 & 2939 & GRSD, BRRI \\
\hline Chini Sagar (2) & 245 & Mymensingh & Black TAPL-554 & 2947 & GRSD, BRRI \\
\hline Meny & 288 & Gaibandha & Straw TAPL-500 & 2898 & GRSD, BRRI \\
\hline Tilkapur & 296 & Gaibandha & Dubsail & 4840 & Satkhira \\
\hline Binnaphul & 315 & Gaibandha & Duksail & 2028 & Satkhira \\
\hline Kalobhog & 318 & Gaibandha & Khaskani & 4341 & Jashore \\
\hline Jabsiri & 331 & Gaibandha & Khazar & 4921 & Iran \\
\hline Kalgochi & 352 & Gaibandha & Basmati sufaid106 & 4498 & Pakistan \\
\hline Chinisakkor & 387 & Rajshahi & BR5 & 4343 & GRSD, BRRI \\
\hline Chiniatob & 399 & Rajshahi & BRRI dhan34 & 7093 & GRSD, BRRI \\
\hline Noyonmoni & 461 & Rajshahi & BRRI dhan37 & 7094 & GRSD, BRRI \\
\hline Saubail & 873 & Sylhet & BRRI dhan38 & 7095 & GRSD, BRRI \\
\hline Chinniguri & 1880 & Kishoreganj & BRRI dhan50 & 6882 & GRSD, BRRI \\
\hline Kalomala & 1886 & Kishoreganj & Khasa Mukpura & 7586 & Khagrachhari \\
\hline Begunmala & 1896 & Kishoreganj & Uknimodhu & 298 & Gaibandha \\
\hline Gopalbhog & 1938 & Kishoreganj & Bawaibhog-2 & 301 & Gaibandha \\
\hline Tulsimoni & 1980 & Jamalpur & Chiniatob-2 & 398 & Rajshahi \\
\hline Jirabuti & 1984 & Mymensingh & Tilokkachari & 758 & Chittagong \\
\hline Khirshabuti & 1996 & Tangail & Begunbichi-2 & 508 & Rangpur \\
\hline Rajbut & 1999 & Tangail & Chinairri & 764 & Chottagram \\
\hline Soru kamina & 2015 & Satkhira & Bhatir chikon & 774 & Chittagong \\
\hline Kamini soru & 2027 & Satkhira & Gordoi & 1908 & Kishoreganj \\
\hline Doiarguru & 2037 & Khulna & Dolagocha & 451 & Rajshahi \\
\hline Premful & 2041 & Satkhira & Kalonunia & 537 & Rangpur \\
\hline Begun bichi & 2073 & Kishoreganj & Dhan chikon & 538 & Dinajpur \\
\hline Elai & 2423 & Dhaka & Badshabhog-2 & 03 & Dhaka \\
\hline Gua masuri & 3666 & Sherpur & Thakurbhog-2 & 872 & Sylhet \\
\hline Luina & 3676 & Netrokona & Khuti chikon & 4107 & Cumilla \\
\hline Lal Soru & 4135 & Dinajpur & Sunduri samba & 4803 & Rajshahi \\
\hline Chini Kanai & 4356 & Khulna & Basmati & 4754 & Barguna \\
\hline Kalijira (short grain) & 4357 & Khulna & Basmati 37 & 4491 & India \\
\hline Rajbhog & 4360 & Khulna & Basnatu sufaid 187 & 4499 & Pakistan \\
\hline Philliphine kataribhog & 4365 & Dinajpur & Tulsimala-2 & 7342 & Sherpur \\
\hline Baoibhog & 4813 & Kurigram & Chinisail & 7343 & Sherpur \\
\hline Baoijhaki & 4826 & Dinajpur & Malshira & 7347 & Sherpur \\
\hline Jirabhog(Bolder) & 4828 & Dinajpur & Sadagura & - & Khagrachhari \\
\hline Chinigura & 4867 & Mymensingh & Modhumadab & 7352 & Habigang \\
\hline Tulsimala & 4870 & Mymensingh & Parbatjira & 7351 & Habigang \\
\hline Bashmati 370 & 4904 & Pakistan & Chinikanai-2 & 7350 & Dinajpur \\
\hline Uknimodhu & 5083 & Rangpur & Meedhan & 7537 & Habiganj \\
\hline Ranisalut & 5286 & Khulna & Gobindhabhog & - & Jessore \\
\hline Jira dhan & 5313 & Khulna & Kataribhog & 7082 & Dinajpur \\
\hline Gandhakusturi & 5319 & Bagerhat & Fulkari & 7531 & Habiganj \\
\hline Sakkorkhora & 5347 & Barguna & BU Dhan2R & 7413 & GRSD, BRRI \\
\hline Badshabhog & 5349 & Bagerhat & Padmabhog & 4812 & Kurigram \\
\hline Jirakatari & 5975 & Dinajpur & Dudsail & 4840 & Satkhira \\
\hline Desikatari & 5978 & Dinajpur & Sakkorkhana & 4761 & Barguna \\
\hline Thakurbhog & 5983 & Sylhet & Maloti & 169 & Tangail \\
\hline Tulsimaloty & 6638 & Tangail & Bashful & 4215 & Kishoreganj \\
\hline Raduni pagal & 6711 & Rajshahi & KalijiraTAPL-64 & 2492 & GRSD, BRRI \\
\hline Sugandhi dhan & 7063 & Nawabganj & OvalTAPL-2990 & 2990 & GRSD, BRRI \\
\hline Kalijira (long grain) & 4358 & Khulna & KalijiraTAPL-68 & 2496 & GRSD, BRRI \\
\hline Jesso balam TAPL-25 & 2454 & GRSD, BRRI & KalijiraTAPL-74 & 2501 & GRSD, BRRI \\
\hline Dakshahi & 983 & Khulna & Kalobakri & 2108 & Narsingdi \\
\hline Hatisail TAPL-101 & 2528 & GRSD, BRRI & & & \\
\hline
\end{tabular}




\section{Aroma test}

Aroma was detected by sniffing and was scored as non-scented, lightly scented, and scented following 1.7\% $\mathrm{KOH}$ based method (Sood and Siddiq, 1978).

\section{Statistical analysis}

Twenty-eight qualitative data were transformed to binary form described by Sneath and Sokal (1973). For qualitative traits, the presence and absence of the different variants were scored as 1 and 0 respectively. The data analysis was done using the NTSYSpc version 2.2 (Rohlf, 2002 ).

\section{RESULTS AND DISCUSSION}

\section{Qualitative traits characterization}

Agro-morphological characterization is an important activity to evaluate the utilization of the germplasm collection in a genebank (Islam et al., 2018a). The diversity in crop varieties is essential for agricultural development for increasing food production; poverty alleviation and promoting economic growth. The present study was aimed at identifying distinct qualitative traits for aromatic rice germplasm. Polymorphism was found in 25 of the 28 qualitative traits studied; the nonpolymorphic traits were of ligule colour, ligule shape and auricle colour (Table 2). Figure 1 presents variation in grain morphology of some aromatic rice germplasm. Among the 113 aromatic germplasm, $87.61 \%$ showed blade pubescence, $97.35 \%$ green blade colour, $95.58 \%$ green basal leaf sheath colour, $96.46 \%$ horizontal leaf angle, $95.58 \%$ pale green of collar colour, $94.69 \%$ has well exerted panicle and $88.49 \%$ has white colour of stigma. The present study results reveal that all aromatic rice germplasm have the same ligule colour, shape and auricle. Also the variability in most of the observed qualitative traits of aromatic rice germplasm was exhibited in our study. Similar studies were also reported by other researchers (Ahmed et al., 2016; Mau et al.,
2017; Akter et al., 2017 and Islam et al., 2017). However, Islam et al. (2018a) found that variation for leaf blade colour, lemma-palea colour, apiculus colour, lemma-palea pubescence and seed coat colour in similar named of aromatic rice landraces. Similarly, genetic variability in Kartiksail rice accessions of Bangladesh using qualitative agromorphological character was also reported by Ahmed et al. (2015).

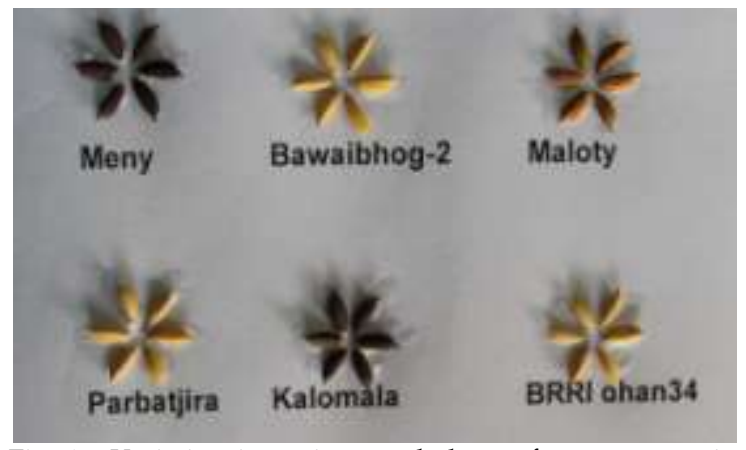

Fig. 1. Variation in grain morphology of some aromatic rice germplasm.

Cluster analysis based on 28 qualitative traits The dendrogram were constructed on the basis of data generated from the 28 qualitative traits. Genetic distance ranged from 0.00 to 2.17 which revealed significant differences among test germplasm. The 113 aromatic germplasm were grouped into 10 clusters. As evident from Figure 2 and Table 3, the highest numbers of germplasm (96) were found in cluster IXd, 2 was found in cluster III, IV and VI, 3 were in IXc and the lowest number of genotypes (1) in cluster I, II,V, VII, VIII, IXa, IXb and X, respectively. Cluster IX consisted of four subclusters (IXa, IXb, IXc and IXd). Cluster IX subclusters IXa, IXb, IXc and IXd consisted of 1, 1, 3 and 96 aromatic germplasm, respectively. Similarly, Hossain (2008) observed 10 clusters by using UPGMA clustering method in 78 aromatic and fine grain landraces of rice genotypes. Two germplasm namely Kalgochi and Buchi in cluster IV were found similarity in 26 of the 28 qualitative traits studied and had very long awn (>20 mm). Bashful, Khazar, 
Table 2. Classification of aromatic germplasm based on 28 qualitative characters.

\begin{tabular}{|c|c|c|c|c|}
\hline Character & Classification & Frequency & Number of aromatic germplasm & Frequency $\%$ \\
\hline \multirow{7}{*}{$\begin{array}{l}\text { Blade } \\
\text { pubescence }\end{array}$} & 01. Glabrous & 2 & 105,95 & 1.77 \\
\hline & 02. Intermediate & 12 & $20,30,67,73,104,106,107,109,110,111,112,113$ & 10.62 \\
\hline & 03. Pubescent & 99 & $1,2,3,4,5,6,7,8,9,10,11,12,13,14,15,16,17,18,19,21,22,23,2$ & 87.61 \\
\hline & & & $4,25,26,27,28,29,31,32,33,34,35,36,37,38,39,40,41,42,43,4$ & \\
\hline & & & $4,45,46,47,48,49,50,51,52,53,54,55,56,57,58,59,60,61,62,6$ & \\
\hline & & & $3,64,65,66,68,69,70,71,72,74,75,76,77,78,79,80,81,82,83,8$ & \\
\hline & & & $\begin{array}{l}4,85,86,87,88,89,90,91,92,93,94,96,97,98,99,100,101,102 \\
103,108\end{array}$ & \\
\hline \multirow[t]{7}{*}{ Blade colour } & 01. Pale green & 01 & 84 & 0.88 \\
\hline & 02. Green & 110 & $1,2,3,4,5,6,7,8,9,10,11,12,13,14,15,16,17,18,19,20,21,22,2$ & 97.35 \\
\hline & & & $3,24,25,26,27,28,29,30,31,32,33,34,35,36,37,38,39,40,41,4$ & \\
\hline & & & $2,43,44,45,46,47,48,49,50,51,52,54,55,56,57,58,59,60,61,6$ & \\
\hline & & & $2,63,64,65,66,67,68,69,70,71,72,73,74,75,76,77,78,79,80,8$ & \\
\hline & & & $\begin{array}{l}1,82,83,85,86,87,88,89,90,91,92,93,94,95,96,97,98,99,100, \\
101,102,104,105,106,107,108,109,110,111,112,113\end{array}$ & \\
\hline & 03. Dark green & 02 & 53,103 & 1.77 \\
\hline \multirow{7}{*}{$\begin{array}{l}\text { Leaf sheath: } \\
\text { anthocyanin } \\
\text { colour }\end{array}$} & 01. Absent & 108 & $1,2,3,4,5,6,7,8,9,10,11,12,13,14,15,16,17,18,19,21,22,23,2$ & 95.58 \\
\hline & & & $4,25,26,27,28,29,30,31,32,33,34,35,36,37,38,39,40,41,42,4$ & \\
\hline & & & $3,44,45,46,47,48,49,50,51,52,53,54,55,56,57,58,59,60,61,6$ & \\
\hline & & & $2,63,64,65,67,68,69,70,71,72,73,74,75,76,77,78,79,80,81,8$ & \\
\hline & & & $2,83,84,85,88,89,90,91,92,93,94,95,96,97,98,99,100,101,1$ & \\
\hline & & & $02,103,104,105,106,107,109,110,111,112,113$ & \\
\hline & 09. Present & 05 & $20,66,86,87,108$ & 4.42 \\
\hline \multirow{8}{*}{$\begin{array}{l}\text { Basal leaf } \\
\text { sheath colour }\end{array}$} & 01. Green & 108 & $1,2,3,4,5,6,7,8,9,10,11,12,13,14,15,16,17,18,19,21,22,23,2$ & 95.58 \\
\hline & & & $4,25,26,27,28,29,30,31,32,33,34,35,36,37,38,39,40,41,42,4$ & \\
\hline & & & $3,44,45,46,47,48,49,50,51,52,53,54,55,56,57,58,59,60,61,6$ & \\
\hline & & & $2,63,64,65,67,68,69,70,71,72,73,74,75,76,77,78,79,80,81,8$ & \\
\hline & & & $2,83,84,85,88,89,90,91,92,93,94,95,96,97,98,99,100,101,1$ & \\
\hline & & & $02,103,104,105,106,107,109,110,111,112,113$ & \\
\hline & 03. Light purple & 03 & $20,86,87$ & 2.65 \\
\hline & 04. Purple & 02 & 66,108 & 1.77 \\
\hline \multirow[t]{7}{*}{ Leaf angle } & 01. Erect & 03 & $10,59,72,103$ & 3.54 \\
\hline & 05. Horizontal & 110 & $1,2,3,4,5,6,7,8,9,11,12,13,14,15,16,17,18,19,20,21,22,23,2$ & 96.46 \\
\hline & & & $4,25,26,27,28,29,30,31,32,33,34,35,36,37,38,39,40,41,42,4$ & \\
\hline & & & $3,44,45,46,47,48,49,50,51,52,53,54,55,56,57,58,60,61,62,6$ & \\
\hline & & & $3,64,65,66,67,68,69,70,71,73,74,75,76,77,78,79,80,81,82,8$ & \\
\hline & & & $3,84,85,86,87,88,89,90,91,92,93,94,95,96,97,98,99,100,10$ & \\
\hline & & & $1,102,104,105,106,107,108,109,110,111,112,113$ & \\
\hline \multirow{10}{*}{$\begin{array}{l}\text { Flag leaf } \\
\text { angle }\end{array}$} & 01. Erect $\left(<30^{\circ}\right.$ & 02 & 72,103 & 1.77 \\
\hline & $\begin{array}{l}\text { 03. Semi erect }(<30- \\
\left.45^{\circ}\right)\end{array}$ & 03 & $10,59,107$ & 2.65 \\
\hline & 05. Horizaontal $(<46-$ & 104 & $1,4,5,6,8,9,11,12,13,14,15,16,17,18,19,20,21,22,23,24,25,2$ & 92.04 \\
\hline & $\left.90^{\circ}\right)$ & & $6,27,28,29,30,31,32,33,34,35,37,38,39,40,41,42,43,44,45,4$ & \\
\hline & & & $6,47,48,49,50,51,52,53,54,55,56,57,58,60,61,62,63,64,65,6$ & \\
\hline & & & $6,67,68,69,70,71,73,74,75,76,77,78,79,80,81,82,83,84,85,8$ & \\
\hline & & & $6,87,88,89,90,91,92,93,94,95,96,97,98,99,100,101,102,104$ & \\
\hline & & & $, 105,106,108,109,110,111,112,113$ & \\
\hline & 07. Descending & & & 3.54 \\
\hline & $\left(>90^{\circ}\right)$ & 04 & $2,3,7,36$ & \\
\hline
\end{tabular}

Agro-morphological Characterization of Bangladeshi Aromatic Rice 45 
Table 2. Continued.

\begin{tabular}{|c|c|c|c|c|}
\hline Character & Classification & Frequency & Number of aromatic germplasm & Frequency $\%$ \\
\hline Ligule colour & 01. White & 113 & $\begin{array}{l}1,2,3,4,5,6,7,8,9,10,11,12,13,14,15,16,17,18,19,20,21,22,2 \\
3,24,25,26,27,28,29,30,31,32,33,34,35,36,37,38,39,40,41,4 \\
2,43,44,45,46,47,48,49,50,51,52,53,54,55,56,57,58,59,60,6 \\
1,62,63,64,65,66,67,68,69,70,71,72,73,74,75,76,77,78,79,8 \\
0,81,82,83,84,85,86,87,88,89,90,91,92,93,94,95,96,97,98,9 \\
9,100,101,102,103,104,105,106,107,108,109,110,111,112, \\
113\end{array}$ & Nil \\
\hline Ligule shape & 02. 2-cleft & 113 & $\begin{array}{l}1,2,3,4,5,6,7,8,9,10,11,12,13,14,15,16,17,18,19,20,21,22,2 \\
3,24,25,26,27,28,29,30,31,32,33,34,35,36,37,38,39,10,41,4 \\
2,43,44,45,46,47,48,49,50,51,52,53,54,55,56,57,58,59,60,6 \\
1,62,63,64,65,66,67,68,69,70,71,72,73,74,75,76,77,78,79,8 \\
0,81,82,83,84,85,86,87,88,89,90,91,92,93,94,95,96,97,98,9 \\
9,100,101,102,103,104,105,106,107,108,109,110,111,112, \\
113\end{array}$ & Nil \\
\hline Collar colour & 01. Pale green & 108 & $\begin{array}{l}2,3,4,5,6,7,8,9,11,12,13,14,15,16,17,18,19,20,21,22,23,24, \\
25,26,27,28,29,30,31,32,33,34,35,36,37,38,39,40,41,42,43, \\
44,45,46,47,48,49,50,51,52,53,54,55,56,57,58,59,60,61,62, \\
63,64,65,67,68,69,70,71,72,73,74,75,76,77,78,79,80,81,82, \\
83,84,85,86,87,88,89,90,91,92,93,95,96,97,98,99,100,101, \\
102,103,104,105,106,107,109,110,111,112,113\end{array}$ & 95.58 \\
\hline & 03. Purple & 05 & $1,10,66,94,108$ & 4.42 \\
\hline Auricle colour & 01. Pale green & 113 & $\begin{array}{l}1,2,3,4,5,6,7,8,9,10,11,12,13,14,15,16,17,18,19,20,21,22,2 \\
3,24,25,26,27,28,29,30,31,32,33,34,35,36,37,38,39,40,41,4 \\
2,43,44,45,46,47,48,49,50,51,52,53,54,55,56,57,58,59,60,6 \\
1,62,63,64,65,66,67,68,69,70,71,72,73,74,75,76,77,78,79,8 \\
0,81,82,83,84,85,86,87,88,89,90,91,92,93,94,95,96,97,98,9 \\
9,100,101,102,103,104,105,106,107,108,109,110,111,112, \\
113\end{array}$ & Nil \\
\hline \multirow[t]{2}{*}{$\begin{array}{l}\text { Culm } \\
\text { anthocyanin } \\
\text { colour }\end{array}$} & 01. Absent & 110 & $\begin{array}{l}1,2,3,4,5,6,7,8,9,10,11,12,13,14,15,16,17,18,19,21,22,23,2 \\
4,25,26,27,28,29,30,31,32,33,34,35,36,37,38,39,40,41,42,4 \\
3,44,45,46,47,48,49,51,52,53,54,55,56,57,58,59,60,61,62,6 \\
3,64,65,67,68,69,70,71,72,73,74,75,76,77,78,79,80,81,82,8 \\
3,84,85,87,88,89,90,91,92,93,94,95,96,97,98,99,100,101,1 \\
02,103,104,105,106,107,109,110,111,112,113\end{array}$ & 96.46 \\
\hline & 09. Present & 04 & $20,66,86,108$ & 3.54 \\
\hline \multirow[t]{3}{*}{ Culm Angle } & 01. Erect $\left(<30^{0}\right)$ & 33 & $\begin{array}{l}2,3,5,7,12,21,22,27,35,36,40,41,43,44,46,47,48,52,53,57,6 \\
0,61,62,63,64,65,66,72,92,99,102,103,105\end{array}$ & 29.21 \\
\hline & 03. Intermediate & 68 & $\begin{array}{l}4,6,8,9,10,11,13,14,15,16,17,19,20,23,24,25,26,29,30,31,3 \\
2,33,34,37,38,39,42,45,49,51,54,55,56,58,59,68,69,70,71,7 \\
3,74,77,78,79,80,81,82,84,85,86,87,88,89,90,91,93,94,95,9 \\
6,97,98,100,101,104,106,108,110,112\end{array}$ & 60.18 \\
\hline & 05. Open & 12 & $1,18,28,50,67,75,76,83,107,109,111,113$ & 10.62 \\
\hline \multirow[t]{4}{*}{$\begin{array}{l}\text { Internode } \\
\text { colour }\end{array}$} & 01. Green & 89 & $\begin{array}{l}4,5,6,7,8,9,10,11,12,13,14,15,16,17,18,19,21,22,23,24,25,2 \\
6,27,28,29,30,31,32,33,34,35,36,37,38,39,40,41,42,43,44,4 \\
5,46,47,48,49,50,51,52,53,54,55,56,57,58,59,60,61,62,63,6 \\
4,65,68,69,70,71,72,73,74,79,80,81,83,84,86,87,90,92,93,9 \\
4,95,96,98,99,100,101,102,103,106,113\end{array}$ & 78.76 \\
\hline & 02. Light gold & 20 & $\begin{array}{l}\text { 2,3,67,75,76,77,78,82,85,88,89,91,97,104,105,107,109,110 } \\
, 111,112\end{array}$ & 17.71 \\
\hline & 03. Purple lines & 03 & $1,20,108$ & 2.65 \\
\hline & 04. Purple & 01 & 66 & 0.88 \\
\hline Culm & 01. Strong & 03 & $53,72,103$ & 2.65 \\
\hline
\end{tabular}


Table 2. Continued.

\begin{tabular}{|c|c|c|c|c|}
\hline Character & Classification & Frequency & Number of aromatic germplasm & Frequency $\%$ \\
\hline \multirow[t]{4}{*}{ strength } & $\begin{array}{l}\text { 03. Moderately } \\
\text { strong }\end{array}$ & 01 & 104 & 0.88 \\
\hline & 05. Intermediate & 18 & $2,25,43,45,46,60,77,78,79,80,84,95,96,97,102,105,106,110$ & 15.93 \\
\hline & 07. Weak & 68 & $\begin{array}{l}1,3,4,5,6,7,8,9,10,11,13,14,15,16,17,19,20,24,26,27,28,29, \\
30,41,47,52,54,56,58,59,61,63,64,65,66,67,68,69,71,73,74, \\
75,76,81,82,83,85,86,87,88,89,90,91,92,93,94,98,99,100,1 \\
01,107,108,109,111,112,113\end{array}$ & 60.18 \\
\hline & 09. Very weak & 25 & $\begin{array}{l}12,18,21,22,23,31,32,33,34,35,36,37,38,39,40,42,44,48,49 \\
50,51,55,57,62,70\end{array}$ & 22.12 \\
\hline \multirow[t]{3}{*}{ Panicle type } & 01. Compact & 09 & $10,19,20,25,47,59,72,103,110$ & 7.96 \\
\hline & 05. Intermediate & 97 & $\begin{array}{l}1,4,5,6,7,8,9,11,12,13,14,15,16,17,18,21,22,23,24,26,27,28 \\
, 29,30,31,32,33,34,35,36,37,38,39,40,41,42,43,44,45,46,48 \\
499,50,51,52,53,54,55,56,57,58,60,61,62,63,64,65,66,67,68 \\
, 69,70,71,73,74,77,78,79,80,81,82,83,84,85,86,87,88,89,90 \\
, 91,92,93,94,95,96,97,98,99,100,101,102,104,105,106,107, \\
112,113\end{array}$ & 85.84 \\
\hline & 09. Open & 07 & $2,3,75,76,108,109,111$ & 6.19 \\
\hline \multirow[t]{2}{*}{$\begin{array}{l}\text { Secondary } \\
\text { branching }\end{array}$} & 01. Light & 68 & $\begin{array}{l}1,2,3,6,7,8,9,10,12,13,14,15,17,18,21,22,23,25,26,28,29,37 \\
, 38,39,40,41,43,44,45,48,49,50,51,52,53,54,55,57,59,60,61 \\
, 62,63,64,66,67,70,71,72,76,77,79,81,86,89,90,91,101,103 \\
104,106,108,109,110,111,112,113,114\end{array}$ & 59.29 \\
\hline & 02. Heavy & 46 & $\begin{array}{l}4,5,11,16,19,20,24,27,30,31,32,33,34,35,36,42 \\
46,47,56,58,65,68,69,73,74,75,78,80,82,83,84,85,87,88,92 \\
93,94,95,96,97,98,99,100,102,105,107\end{array}$ & 40.70 \\
\hline \multirow[t]{3}{*}{$\begin{array}{l}\text { Panicle } \\
\text { exsertion }\end{array}$} & 01. Well exserted & 107 & $\begin{array}{l}1,2,3,4,5,7,8,9,10,11,12,13,14,15,16,17,18,19,20,21,22,23, \\
24,25,26,27,29,30,31,32,33,34,35,36,37,38,39,40,41,42,43, \\
44,45,46,47,48,49,50,51,52,54,55,56,57,58,59,60,61,64,65, \\
67,68,69,70,71,72,73,74,75,76,77,78,79,80,81,82,83,84,85, \\
86,87,88,89,90,91,92,93,94,95,96,97,98,99,100,101,102,10 \\
3,104,105,106,107,108,109,110,111,112,113\end{array}$ & 94.69 \\
\hline & $\begin{array}{l}\text { 03. Moderately well } \\
\text { exserted }\end{array}$ & 05 & $6,28,53,62,63$ & 4.42 \\
\hline & 05. Just exserted & 01 & 66 & 0.88 \\
\hline \multirow{3}{*}{$\begin{array}{l}\text { Spikelet: } \\
\text { awns in the } \\
\text { spikelet }\end{array}$} & 01. Absent & 78 & $\begin{array}{l}1,2,4,5,6,7,11,12,14,15,16,17,19,20,21,23,25,26,27,28,32,3 \\
3,36,37,39,40,42,43,45,46,50,51,53,55,56,57,58,62,63,64,6\end{array}$ & \\
\hline & & & $\begin{array}{l}5,68,69,72,73,74,75,76,77,78,79,80,82,84,85,86,87,92,93,9 \\
4,95,96,97,98,99,100,101,102,103,104,105,106,107,108,10 \\
9,110,111,112\end{array}$ & 69.03 \\
\hline & 09. Present & 35 & $\begin{array}{l}3,8,9,10,13,18,22,24,29,30,31,34,35,38,41,44,48,49,52,54 \\
59,60,61,62,66,67,70,71,81,83,88,89,90,91,113\end{array}$ & 30.97 \\
\hline \multirow[t]{5}{*}{$\begin{array}{l}\text { Spikelet: } \\
\text { awn length }\end{array}$} & $\begin{array}{l}01 . \text { Very short } \\
(<2 \mathrm{~mm})\end{array}$ & 11 & $8,29,34,38,48,49,62,88,89,90,91$ & 9.73 \\
\hline & 03. Short $(2-5 \mathrm{~mm})$ & 02 & 41,43 & 1.77 \\
\hline & $\begin{array}{l}\text { 05. Medium (5-10 } \\
\mathrm{mm})\end{array}$ & 07 & $3,30,35,44,54,70,81$ & 6.19 \\
\hline & 07. Long (11-20 mm) & 02 & 31,67 & 1.77 \\
\hline & $\begin{array}{l}\text { 09. Very long } \\
(>20 \mathrm{~mm})\end{array}$ & 14 & $9,10,13,18,22,24,52,59,60,61,66,71,113$ & 11.50 \\
\hline \multirow[t]{2}{*}{$\begin{array}{l}\text { Distribution } \\
\text { of awning }\end{array}$} & 01. Tip only & 17 & $3,8,29,30,34,35,38,41,44,48,49,62,70,81,88,89,90,91$ & 15.04 \\
\hline & 03. Upper half only & 06 & $13,24,31,54,67,83$ & 5.30 \\
\hline
\end{tabular}


Table 2. Continued.

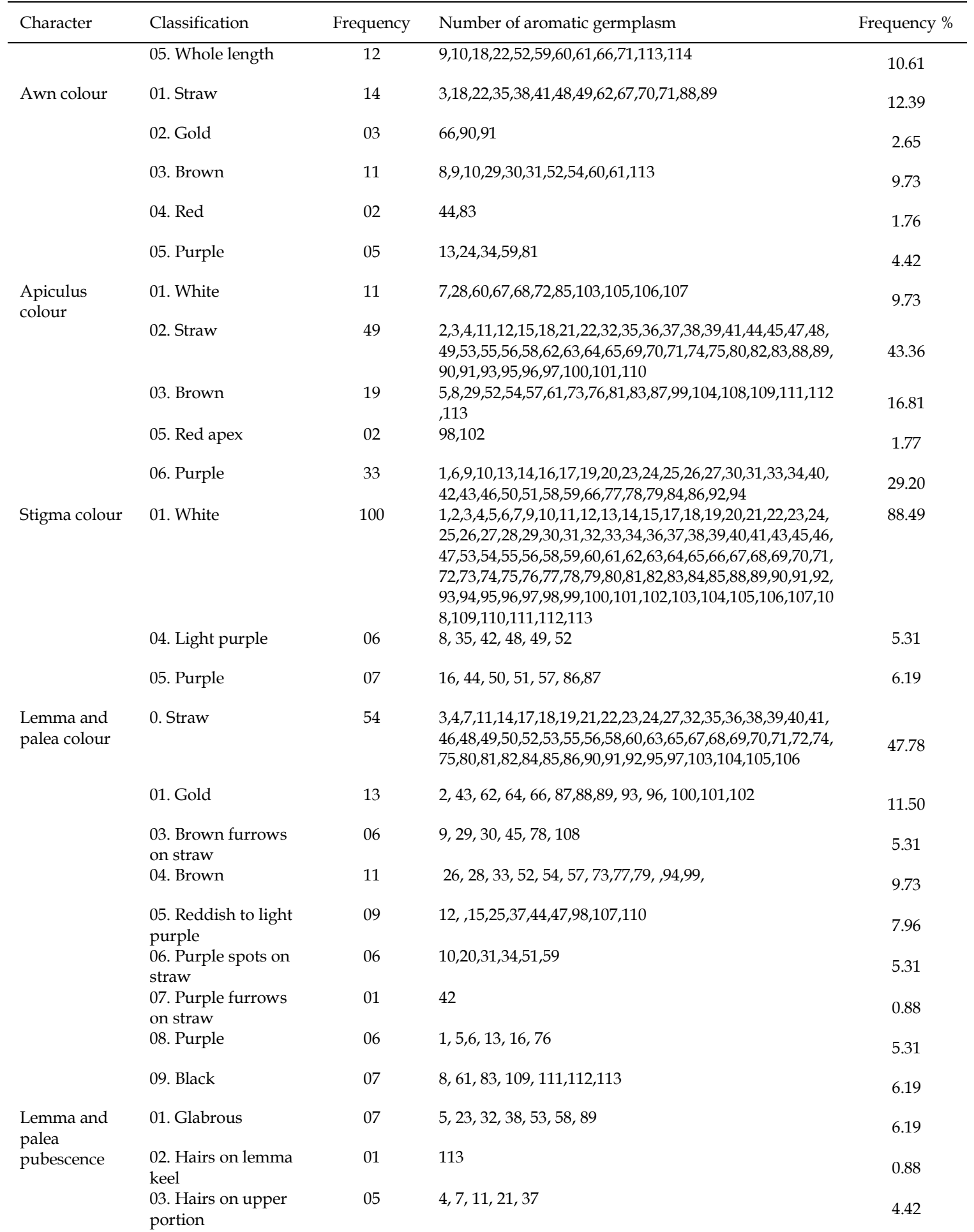


Table 2. Continued.

\begin{tabular}{|c|c|c|c|c|}
\hline Character & Classification & Frequency & Number of aromatic germplasm & Frequency $\%$ \\
\hline \multirow{5}{*}{$\begin{array}{l}\text { Seed coat } \\
\text { (bran) colour }\end{array}$} & 04. Short hairs & 75 & $\begin{array}{l}1,2,3,6,8,9,13,14,15,16,17,18,19,20,22,24,25,26,27,28,29,3 \\
0,31,33,34,35,36,39,10,41,42,44,46,47,48,49,50,51,52,54,5 \\
5,56,65,66,68,69,70,72,74,75,77,78,79,80,81,82,83,84,85,8 \\
6,87,88,92,93,95,97,100,101,102,103,104,105,106,107, \\
110\end{array}$ & 66.37 \\
\hline & 05. Long hairs & 25 & $\begin{array}{l}10,12,43,45,57,59,60,61,62,63,64,67,71,73,76,90,91 \\
94,96,98,99,108,109,111,112\end{array}$ & 22.12 \\
\hline & 01. White & 79 & $\begin{array}{l}2,3,7,9,10,11,14,17,18,19,20,21,22,23,24,25,26,30,31,32,3 \\
3,34,35,36,37,38,39,40,41,42,43,44,46,47,48,49,50,53,55,5 \\
6,57,58,59,60,61,62,63,64,65,66,67,68,69,70,71,72,75,80,8 \\
2,84,85,86,87,88,89,91,92,93,94,96,97,98,101,103,105,106 \\
, 107,110,113\end{array}$ & 69.91 \\
\hline & 02. Light brown & 32 & $\begin{array}{l}1,4,5,6,8,12,13,15,16,27,29,45,51,52,54,73,74, \\
76,77,78,79,81,83,90,95,99,100,102,104,109,111,112\end{array}$ & 28.31 \\
\hline & 05. Red & 02 & 28,108 & 1.76 \\
\hline \multirow{3}{*}{$\begin{array}{l}\text { Leaf } \\
\text { senescence }\end{array}$} & 01. Late and slow & 03 & $45,50,61$ & 2.65 \\
\hline & 05. Intermediate & 13 & $9,10,14,43,55,58,60,62,63,72,80,103,113$ & 11.50 \\
\hline & 09. Early and fast & 97 & $\begin{array}{l}1,2,3,4,5,6,7,8,11,12,13,15,16,17,18,19,20,21,22,23,24,25, \\
26,27,28,29,30,31,32,33,34,35,36,37,38,39,40,41,42,44,46, \\
47,48,49,51,52,53,54,56,57,59,64,65,66,67,68,69,70,71,73, \\
74,75,76,77,78,79,81,82,83,84,85,86,87,88,89,90,91,92,93, \\
94,95,96,97,98,99,100,101,102,104,105,106,107,108,109,1 \\
10,111,112\end{array}$ & 85.84 \\
\hline \multirow{3}{*}{$\begin{array}{l}\text { Decorticated } \\
\text { grain: Scent } \\
\text { (aroma) }\end{array}$} & 0. Non scented & 12 & $28,29,45,50,53,56,64,66,81,82,86,88$ & 10.62 \\
\hline & 01. Lighty scented & 35 & $\begin{array}{l}1,2,3,6,7,23,24,25,27,37,38,43,67,72,83,84,87,89,90,91,92, \\
93,94,95,96,97,98,99,100,102,103,104,105,107,110\end{array}$ & 30.97 \\
\hline & 02. Scented & 66 & $\begin{array}{l}4,5,8,9,10,11,12,13,14,15,16,17,18,19,20,21,22,26,30,31,3 \\
2,33,34,35,36,39,40,41,42,44,46,47,48,49,51,52,54,55,57,5 \\
8,59,60,61,62,63,65,68,69,70,71,73,74,75,76,77,78,79,80 \\
85,101,106,108,109,111,112,113\end{array}$ & 58.41 \\
\hline
\end{tabular}

Sugandhi dhan, Jirabuti, Elai, Dhan chikon, Malshira and Sakor were clustered in indivisul group I, II, V,VII, VIII and sub-cluster IXa, IXb and $\mathrm{X}$ respectively. The germplasm like as Jirabuti, Khazar, Thakurbhog, Khuti Chikon and Bashful had special qualitative traits such as anthocyanin colour of leaf sheath. On the other hand, Jirabuti, Khazar, Thakurbhog and Bashful had anthocyanin colour of culm nodes except Khuti chikon. Cluster IX, sub-clusters IXa, IXb, IXc and IXd were sub-grouped according to their special distinctive qualitative traits and germplasm in the different sub-clusters were closely distant to each other. In general, most of the germplasm fall in fourth major sub-cluster IXd contained 96 aromatic rice germplasm. Basal leaf sheath colour, leaf angle, flag leaf angle, clum angle, culm strength, panicle type and leaf senescence of these 96 germplasm were very close. Therefore, all closely related germplasm were found in same sub-cluster IXd. Parikh et 
al. (2012) also found that majority of the germplasm to possess green basal leaf sheath colour $(84.5 \%)$, green leaf blade colour (86.8\%), green collar colour $(97.3 \%)$, white ligule colour $(94.7 \%)$, light green auricle colour (97.3\%), white apiculus colour (53.9\%), white stigma colour (94.7\%) and awnless (72.3\%) in 71 aromatic rice germplasm. Moreover, most of the cultivated aromatic rice genotypes are photosensitive and taller types having yield potentiality of 2-3 $\mathrm{t} \mathrm{ha}^{-1}$ and grown during $\mathrm{T}$. Aman season in the rainfed low land ecosystem in Bangladesh (Islam et al., 2016). The two germplasm namely Dhan chikon and Malshira were found in sub-cluster IXa and $\mathrm{IXb}$ respectively. Ranisalut, Gandhakusturi, Thakurbhog were found in sub-cluster IXc. Interestingly, BRRI dhan50 and BU dhan2R, which have similiar plant type, yield and grain characters, placed in the same cluster III. Among the other cluster, Sakor, a slight aromatic rice germplasm grown mainly in Mymensingh region and with no relation to the other germplasm, formed a single cluster. A study conducted by Bisne and Sarawgi (2008) to characterize 32 aromatic rice accessions of Badshahbhog group from Indira Gandhi Krishi Vishwavidyalaya (IGKV), Raipur, Chhattisgarh, germplasm, found the highest variation among accessions for the traits leaf blade colour, lemma and palea colour, apiculus colour, and lemma and palea pubescence.

Moreover, aroma evaluation revealed that 67 germplasm were scented, 34 were lightly scented, while the rest 12 germplasm were non-scented (Table 4). For example, local variety including aromatic rice germplasm occupied about $12.16 \%$ of the rice growing area in Bangladesh (Islam et al., 2016). Among the aromatic rice germplasm, Chinigura is the predominant one that covers more than $70 \%$ farms in the northern districts of Naogaon and Dinajpur. In these districts, $30 \%$ of rice lands were covered by aromatic rice varieties during Aman season. The other important aromatic rice varieties are Kalijira (predominant in Mymensingh) and Kataribhog (predominant in Dinajpur) (Baqui et al., 1997).

\section{Principal co-ordinate analysis (PCoA)}

The three dimensional (3D) graphical views of principal co-ordinate analysis (PCA) showed the spatial distribution of the germplasm. The germplasm namely Bashful, Khazar, Jirabuti, Sakor, Kutichikon, Thakurbhog-2, Black TAPL554, Kalgochi and Buchi were found to be distance from the centroid (Fig. 3) while the rest were close to the centroid. The results indicated that the germplasm that were placed far away from the centroid were more genetically diverse, while the genotypes that were placed near the centroid possessed more or less similar genetic background. Similar findings were also reported by other authors (Siddique et al., 2016a, 2016b). However, centroid may be defined as the vector representing the middle point of the cluster which contained at least one number for each variable. The connecting lines between each germplasm and the centroid represented eigenvectors for the respective germplasm. 


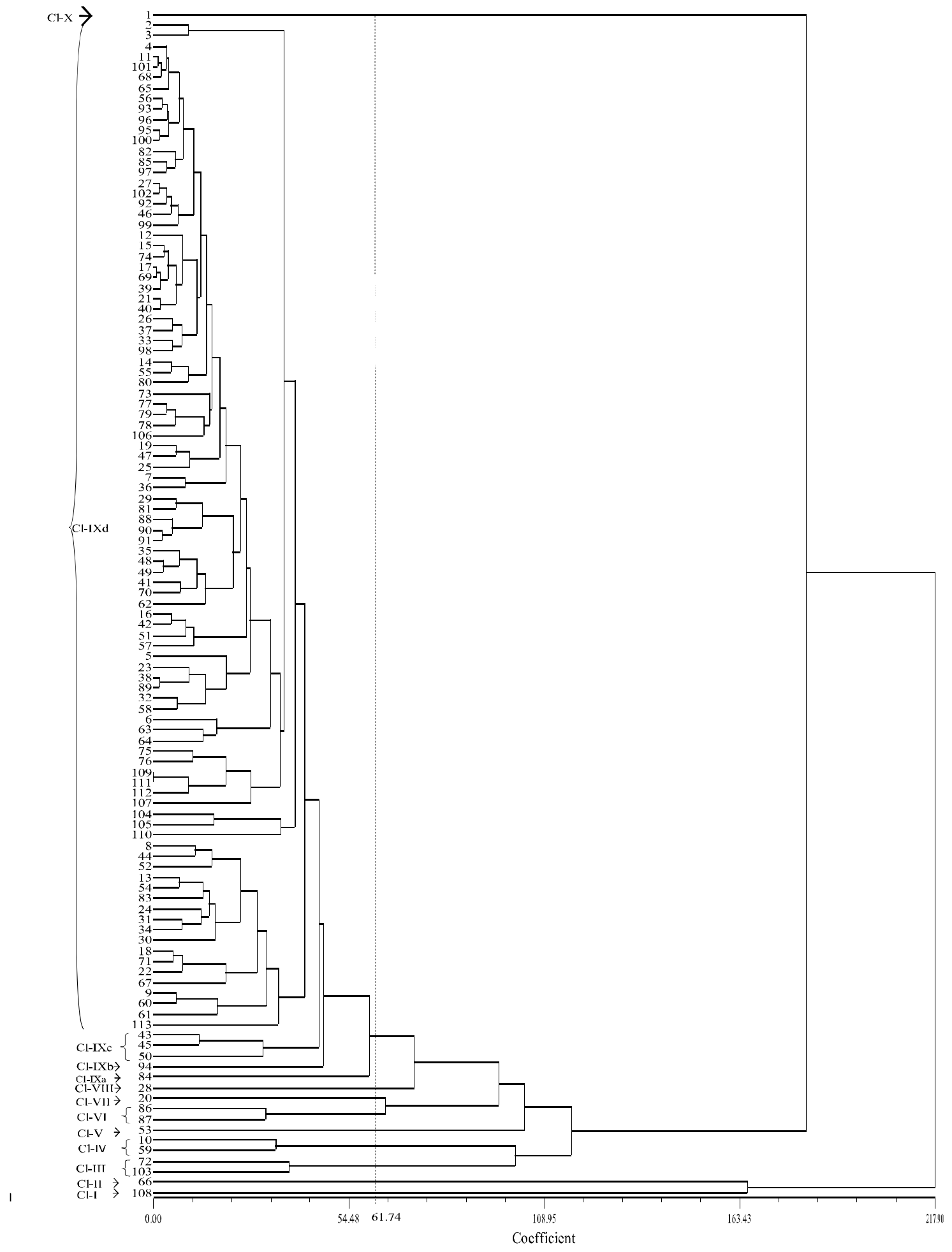

Fig. 2. Dendrogram of 113 test germplasm based on 28 qualitative traits. 
Table 3. Cluster distribution of $\mathbf{1 1 3}$ aromatic germplasm based on $\mathbf{2 8}$ qualitative traits.

\begin{tabular}{|c|c|c|}
\hline Cluster & No. of germplasm & Name of germplasm \\
\hline I & 1 & Bashful \\
\hline II & 1 & Khazar \\
\hline III & 2 & BRRI Dhan50, BU dhan2R \\
\hline IV & 2 & Kalgochi ,Buchi \\
\hline V & 1 & Sugandhi dhan \\
\hline VI & 2 & Thakurbhog-2, Khuti chikon \\
\hline VII & 1 & Jirabuti \\
\hline VIII & 1 & Elai \\
\hline IXa & 1 & Dhan chikon \\
\hline $\mathrm{IXb}$ & 1 & Malshira \\
\hline IXc & 3 & Ranisalut, Gandhakusturi, Thakurbhog \\
\hline IXd & 96 & $\begin{array}{l}\text { Sagardana, Nunia, Chini Sagar (2), Meny, Tilkapur, Binnaphul, Kalobhog, Jabsiri, } \\
\text { Chinisakkor, Chiniatob, Noyonmoni, Saubail, Chinniguri, Kalomala, Begunmala, } \\
\text { Gopalbhog, Tulsimoni, Khirshabuti, Rajbut, Soru kamina, Kamini soru, Doiarguru, } \\
\text { Premful, Begun bitchi, Gua masuri, Luina, Lal Soru, Chini Kanai, Kalijira (short } \\
\text { grain), Rajbhog, Philliphine kataribhog, Baoibhog, Baoijhaki, Jirabhog (Bolder), } \\
\text { Chinigura, Tulsimala, Bashmati 370, Uknimodhu, Jira dhan, Sakkorkhora, } \\
\text { Badshabhog, Jirakatari, Desikatari, Tulsimaloty, Raduni pagal, Kalijira (long grain), } \\
\text { Jesso balam TAPL-25, Dakshahi, Hatisail TAPL-101, Khasa, Awned TAPL-545, Black } \\
\text { TAPL-554, Straw TAPL-554, Dubsail, Duksail, Khaskani, Basmati sufaid 106, BR5, } \\
\text { BRRI dhan34, BRRI dhan37, BRRI dhan38, Khasa Mukpura, Uknimodhu, } \\
\text { Bawaibhog-2, Chiniatob-2, Tilokkachari, Begunbitchi-2, Chinairri, Bhatir cikon, } \\
\text { Gordoi, Dolagocha, Kalonunia, Badshabhog-2, Sunduri samba, Basmati, Basmati 37, } \\
\text { Basnatu sufaid 187, Tulsimala-2, Chinisail, Sadagura, Modhumadab, Parbatjira, } \\
\text { Chinikanai-2, Meedhan, Gobindhabhog, Kataribhog, Fulkari, Padmabhog, Dudsail, } \\
\text { Sakkorkhana, Maloti, KalijiraTAPL-64, OvalTAPL-2990, KalijiraTAPL-68, } \\
\text { KalijiraTAPL-74, Kalobakri }\end{array}$ \\
\hline
\end{tabular}

$\begin{array}{lll}\mathrm{X} & 1 & \text { Sakor }\end{array}$

Table 4. Classification of aromatic germplasm based on sensory test.

\begin{tabular}{lll}
\hline $\begin{array}{l}\text { Decorticated } \\
\text { grain: scent } \\
\text { aroma }\end{array}$ & $\begin{array}{l}\text { Number of } \\
\text { germplasm }\end{array}$ & Name of germplasm \\
\hline
\end{tabular}

\begin{tabular}{|c|c|c|}
\hline Non scented & 12 & $\begin{array}{l}\text { Elai, Gua masuri, Gandha kusturi, Thakurbhog, Sugandhi dhan, Dakshahi, Duksail, } \\
\text { Khazar, Gordoi, Dolagocha, Thakurbhog-2, Sunduri samba }\end{array}$ \\
\hline Light scented & 34 & $\begin{array}{l}\text { Sakor, Sagardana, Nunia, Tilkapur, Binaphul, Soru Kamina, Kamini soru, } \\
\text { Doiarguru, Begun bichi, Baoi jhaki, Jirabhog (Bolder), Ranisaluit, Basmati sufaid- } \\
\text { 106, BRRI dhan50, Kalonunia, Dhan chikon, Khuti chikon, Basmati- 37, Basnatu } \\
\text { sufaid-187, Tulsimala-2, Chinisail, Malshira, Sadagura, Modhumadab, Parbatjira, } \\
\text { Chinikanai-2, Meedhan, Gobindhabhog, Fulkari, BU Dhan2R, Padmabhog, Dudsail, } \\
\text { Maloti, OvalTAPL-2990 }\end{array}$ \\
\hline
\end{tabular}

Scented

Chini Sagar (2), Meny, Kalobhog, Jabsiri, Kalgochi, Chinisakkor, Chini atob, Noyonmoni, Saubail, Kolomala, Chinniguri, Begunmala, Gopalbhog, Tulsimoni, Jirabuti, Khirshaboti, Rajbut, Premful, Luina, Lal Soru, Chini kanai, Kalijira (short grain), Rajbhog, Phillipine kataribhog, Baoibhog, Chinigura, Tulsimala, Bashmati 370, Uknimodhu, Jira dhan, Sakkor khora, Badshabhog, Jirakatari, Desi katari, Tulsimaloty, Radhuni pagal, Kalijira (long grain), Jesso balam, Hatisail, Khasa, Buchi, AwnedTAPL-545, BlackTAPL-554, StrawTAPL-500, Dubsail, Khaskani, BR5, BRRI dhan34, BRRI dhan37, BRRI dhan38, Khasa Mukpura, Uknimodhu, Bawaibhog-2, Chiniatob-2, Tilokkachari, Begunbichi-2, Chinairri, Bhatir cikon, Badshabhog-2, Basmati, Kataribhog, Sakkorkhana, Bashful, KalijiraTAPL-64, Oval TAPL-2990, Kalijira TAPL68, Kalijira TAPL74, Kalobakri 


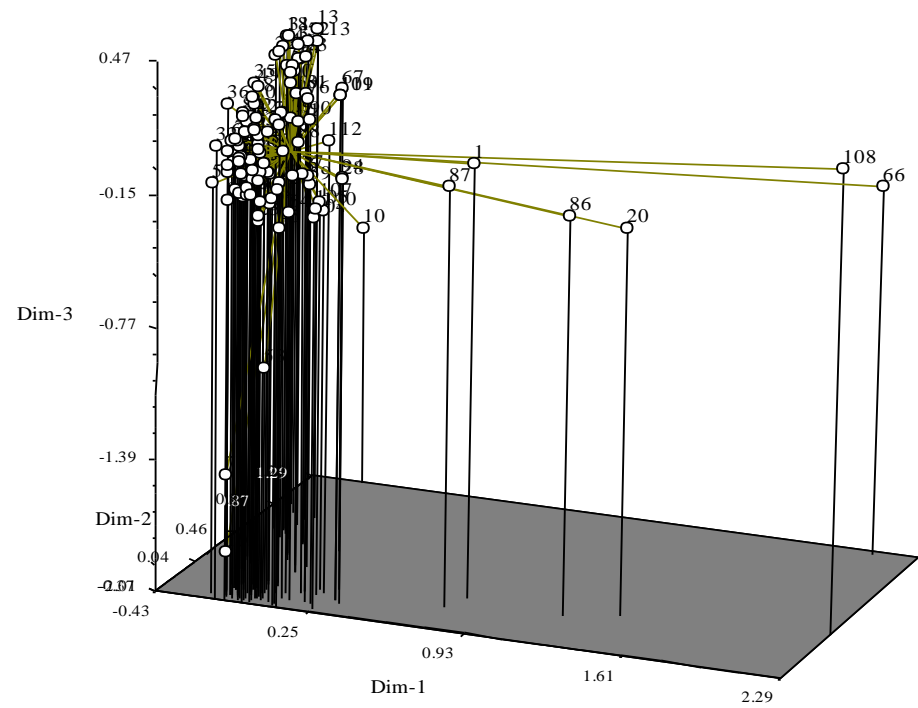

Fig. 3. There-dimensional view of principal co-ordinate analysis (PCoA) of 113 aromatic germplasm with 28 qualitative traits.

\section{CONCLUSIONS}

Traditional aromatic rice germplasm, which is highly chosen by consumers needs to be characterized that can help in varietal development purpose and their conservation (Islam et al., 2018b). No duplicates were identified among the studied germplasm for qualitative traits in the cluster analysis. Aroma is an important trait, has high demand in the global market. The evaluation of aroma showed that 67 germplasm were scented, 34 were lightly scented and 12 were non-scented type. The principal co-ordinate analysis (PCoA) showed the germplasm namely Bashful, Khazar, Jirabuti, Sakor, Kutichikon, Thakurbhog-2, Black TAPL-554 and Kalgochi were found to be the distance from the centroid and they were more genetically diverse. For lemma-palea colour, nine different types were detected while for apiculus colour of grain, six different types were recorded and colour of awn, six different types were observed, suggesting the presence of exclusive variability and unique feature of the traditional short grain aromatic rice germplasm in Bangladesh. Finally, it can be concluded that molecular characterizations of the studied germplasm are required for QTL mapping and validating the presence of candidate genes responsible for valuable characters.

\section{ACKNOWLEDGEMENTS}

The authors are highly grateful to the collaborative research project between the Bangladesh Rice Research Institute (BRRI) and the Bangabandhu Sheikh Mujibur Rahman Agricultural University (BSMRAU) entitled "Genetic enhancement of local rice germplasm towards aromatic hybrid rice variety development in Bangladesh" (2010-2014) funded by the NATP: Phase I of PIU, Bangladesh Agricultural Research Council (BARC) for providing all necessary supports. We also acknowledge $\mathrm{Dr}$ Khandakar $\mathrm{Md}$ Iftekharuddaula, PSO, Plant Breeding Division, BRRI for assisting in statistical analysis. 


\section{REFERENCES}

Ahmed, M S, M K Bashar and A K M Shamsuddin. 2016. Study of qualitative characters of balam rice (Oryza sativa L.) landraces of Bangladesh. Rice Genom. and Genet. 7(1): 1-8.

Ahmed, M S, M Khalequzzaman, M K Bashar. A K M Shamsuddin. 2015. Qualitative agro-morphological character diversity of similar or duplicate named rice (Oryza sativa L.) germplasm of Bangladesh. J. Bio. and Env. Sci. 7 (4): p. 51-61.

Akter, N, H Begum, M Z Islam, M A Siddique and M Khalequzzaman. 2018. Genetic diversity in Aus rice (Oryza Sativa L.) genotypes of Bangladesh. Bangladesh J. Agril. Res. 43(2): 253-266.

Akter, N, M Z Islam, A Bhuiya, M A Siddique and M Khalequzzaman. 2017. Distinctness of 45 irrigated rice (Oryza sativa L.) landraces of Bangladesh through agro-morphological traits. Eco-friendly Agril. J. 10 (10): 100-107.

Bisne, R and A K Sarawgi. 2008. Agro-morphological and quality characterization of Badshahbhog group from aromatic rice germplasm of Chhattisgarh. Bangladesh J. Agril. Res. 33: 479-492.

Bradbury, L M T, S A Gillies, D J Brushett, D L E Waters and R J Henry. 2008. Inactivation of an aminoaldehyde dehydrogenase is responsible for fragrance in rice. Plant Mol. Biol. 68: 439-449.

Bradbury, L M T, T L Fitzgerald, R J Henry, Q Jin and D L E Waters. 2005. The gene for fragrance in rice. Plant Biotech. J. 3:363-370.

Baqui, M A, M E Ham, D Jones, and R Straingfellow. 1997. The export potential of traditional varieties of rice from Bangladesh. Bangladesh Rice Research Institute, Gazipur, Bangladesh.

Glaszmann, J C. 1987. Isozymes and classification of Asian rice varieties. Ther. App. Sci. 74:21-30.

Hossain, M Z. 2008. Genetic diversity for morphophysicochemical characters and microsatellite DNA markers in fine grain and aromatic landraces of rice (Oryza sativa L.). PhD Thesis. Department o f Genetics and Plant Breeding. Bangabandhu Sheikh Mujibur Rahman Agricultural University, Gazipur 1706, Bangladesh. pp.110.

Islam, M Z, M Khalequzzaman, M A Siddique, N Akter, M S Ahmed and M A Z Chowdhury. 2017. Phenotypic characterization of Jhum rice (Oryza sativa L.) landraces collected from Rangamati district in Bangladesh. Bangladesh Rice J. 21 (1): 47-57.

Islam, M Z, M Khalequzzaman, M K Bashar, N A Ivy, M M Haque and M A K Mian. 2016. Variability assessment of aromatic and fine rice germplasm in Bangladesh based on quantitative traits. The Sci. World J. Vol. 2016, Article ID 2796720, http:/ / dx.doi.org/10.1155/2016/2796720.

Islam, M Z, N Akter, T Chakrabarty, A Bhuiya, M A Siddique and M Khalequzzaman. 2018a. Agromorphological characterization and genetic diversity of similar named aromatic rice (Oryza sativa L.) landraces of Bangladesh. Bangladesh Rice J. 22 (1): 45-56.

Islam, M Z , M Khalequzzaman, M K Bashar, N A Ivy, M A K Mian, B R Pittendrigh, M M Haque and M P Ali. 2018b. Variability assessment of aromatic rice germplasm by pheno-genomic traits and population structure analysis. Sci. Reports. 8: 9911, DOI:10.1038/s41598-018-28001-z

Islam, M Z, S Banik, M A Haque, M A Siddiquee, M Khalequzzaman and M A K Mian. 2013. Physicochemical and cooking properties of local aromatic rice germplasm in Bangladesh. Eco-Friendly Agril. J. 6 (10): 243-248.

Mau, Y S, J E R Markus, S S Oematan, A S S Ndiwa, D D Handoko, A Nasution and K Makbul. 2017. Genetic diversity of red and black upland rice accessions from East Nusa Tenggara, Indonesia as revealed by agro morphological characters. Bio-diversitas. 18 (1):197-211.

Morishima, H. 1984. Wild plant and demonstration. In: Biology of Rice (S. Tsunoda and N. Takahashi, Eds.). Japan Sci. Soc. Press, Tokyo. pp. 3-30.

Nascimento, W F, E F Silva and E A Veasey. 2011. Agromorphological characterization of upland rice accessions. Sci. Agric. 68(6): 652-60.

Parikh M., Motiramani N.K., Rastogi N.K., and Sharma B., 2012, Agro-morphological characterization and assessment of variability in aromatic rice germplasm, Bangladesh J. Agril. Res., 37(1): 1-8, http:/ / dx.doi.org/10.3329/bjar.v37i1.11168

Rohlf, F. 2002. NTSYS-pc: Numerical taxonomy and multivariate analysis system. 2.2 edn. Department of Ecology and Evaluation, State university of NY, Stony Brook.

Shahidullah, S M, M M Hanafi, M Ashrafuzzaman, M R Ismail and A Khair. 2009. Genetic diversity in grain quality and nutrition of aromatic rices. African J. Biotech. 8 (7): 1238-1246.

Siddique, M A, M Khalequzzaman M M Islam, Kaniz Fatema and M A Latif. 2016a. Molecular characterization and genetic diversity in geographical indication (GI) rice (Oryza sativa L.) cultivars of Bangladesh. Braz. J. Bot. DOI 10.1007/s40415-016-0271-1

Siddique, M A, M Khalequzzaman, K Fatema, M Z Islam, M M Islam and M A Z Chowdhury. 2016b. Molecular characterization and genetic diversity of Aman rice (Oryza sativa L.) landraces in Bangladesh. Bangladesh Rice J. 20 (2): 1-1.

Singh, R K, U S Singh and G. S. Khush. 2000. Aromatic rices. Oxford and IBH Publishing Co, New Delhi, India. p. 292.

Sneath, P H and Sokal. 1973. Numerical taxonomy: The principles and practice of numerical classification. Freeman WH and Company, Sanfrancisco. USA

Sood, B C and E A Siddiq. 1978. A rapid technique for scent determination in rice. Indian J. Genet. and Plant Breed. 38: 268-271. 